\title{
Marcin Gołaszewski: Vom konservativen Schriftsteller zum inneren Emi- granten. Ernst Wiechert - Eine Fallstudie. Wydawnictwo Uniwersytetu Łódzkiego, Łódź 2017, 579 S.
}

\section{DOI: $10.19195 / 0435-5865.144 .26$}

Schon der Titel des Buches ist irreführend: Vom konservativen Schriftsteller zum inneren Emigranten. Da kann man sich die Frage stellen, was das Adjektiv „konservativ“ für den Verf. bedeutet. Handelt es sich um den Vertreter eines ideologischen Konzeptes, das sich in seiner Homogenität nur widerwillig auf einen gemeinsamen ideologischen Nenner bringen lässt, oder aber um einen Kampfbegriff mit intendiert negativen Konnotationen, die im Widerspruch zum „,inneren Emigranten“ stünden? Das Problematische bei Wiechert wie bei den meisten inneren Emigranten war, dass sie geradezu in Reinkultur den Konservatismus (im Sinne von Gebundenheit an die meist religiöse Tradition) vertraten. Gerade die vor dem „Dritten Reich“ erfolgte „Wende“ Wiecherts war von einer Hinwendung zu den religiösen Werten geprägt, was nicht nur den Inhalt, sondern auch Sprache und Erzählform des Wiechertschen Spätwerkes beeinflusste. Wenn man dazu noch genauer hinschaut, dann wird man in der Zeit nach 1945 manche Aussagen finden, die eindeutig dem konservativ-revolutionären Komplex zuzuordnen sind, wie beispielsweise im Text Der reiche Mann und der arme Lazarus, in dem er Deutschland „Kultur“ und Amerika „Zivilisation“ zuschreibt, anknüpfend an die gängigen Wertungen eines Oswald Spengler (357). Also auch in den späten 40er Jahren sind bei Wiechert nicht nur konservative, sondern konservativ-revolutionäre Wertungen und Unterscheidungen zu finden. Wie der Verf. die Komplexität dieser ideologischen Welten zu kaschieren sucht, erklärt sich zum Teil aus seinen methodischen Voraussetzungen.

Der Verf. glaubt dem Autor, der in seinen autobiographischen Büchern eindeutig an der eigenen Legende von einer Loslösung vom Nationalsozialismus arbeitete, was besonders deutlich in seiner hier umfangreich zitierten Autobiographie „Jahre und Zeiten“ sichtbar wird. Gołaszewski glaubt an den linearen Prozess einer Befreiung vom „konservativen" Gedankengut (konservativ wohl im Sinne der konservativen Revolution), was in seiner mehrere Male wiederholten These anklingt: „Die Phase der inneren Auseinandersetzung mit dem Ersten Weltkrieg und mit den völkisch-nationalen Ideen und Idealen dauert bei Ernst Wiechert bis Anfang der 1930 Jahre.“ (S. 182, siehe dann auch S. 22, auch wie ein Mantra S. 349). Da fragt man sich mit Fug und Recht, warum Wiecherts krasseste völkische Phantasien, wie sie im „Totenwolf“ zum Ausdruck kommen, noch zwei Mal in Hitlers Reich erschienen sind $(1935,1940)$ und - eine für den forschenden Philologen weit bedeutendere Frage - ist die Edition im Rahmen der sämtlichen Werke, die 1957 bei Desch erschienen sind, textgleich mit der Erstausgabe des Buches aus dem Jahr 1924? Für den „Totenwolf" kann ich die Frage aufgrund meiner Autopsie bejahen, für andere Werke der ersten Zeit nicht. Warum setzt sich der Verf. mit diesen philologischen Grundproblemen nicht auseinander, sondern nimmt als seine Quelle auch bei der Betrachtung der Publizistik Wiecherts gerade diese zehnbändige Ausgabe?

Diese posthume Nachkriegsedition kann man als eine Weigerung Wiecherts verstehen, große Teile des eigenen Werkes verdrängen zu wollen, was andere „,innere Emi-

\footnotetext{
* Die Ziffern in Klammern beziehen sich auf diese Ausgabe.
} 
granten“" sehr gerne getan haben. Was macht man aber mit der Strategie des Grote-Verlages, der die beiden oben erwähnten Editionen vom Totenwolf im „Dritten Reich“ zustande brachte? Sind sie als eine nationale oder gar nationalsozialistische „Legitimation" des langsam in die Religiosität abdriftenden Dichters zu verstehen? Oder aber ging es einfach um neue Auflagen sowie ums Geld? Auf jeden Fall scheint es, dass der Verf. einer Interpretationslinie unterliegt, die das Wiechertsche Werk als eine Kontinuität der Entwicklung zum Guten, zum Positiven, zum Humanen, eben zum „Nichtkonservativen“ wahrzunehmen glaubt. Die dichterische Praxis besagt allerdings etwas anderes: nämlich ein enormes Schwanken zwischen einer Akzeptanz des „Dritten Reiches“ und einer Auflehnung gegen die NS-Ideologie bei gleichzeitiger Beibehaltung der eigenen existenziellen Positionen, was gerade im Rahmen einer konservativen Weltanschauung und Poetik möglich war. Mit anderen Worten: Wiechert hat auf seine konservativ-revolutionären Positionen insofern verzichtet, als er das Revolutionäre abschwächte und sich dem traditionellen Konservatismus zuwandte. Allerdings ist dieser Konservatismus durch Spuren der früheren Ideologie genügend durchsetzt, um die Bewunderung eines Hans Frank, des Generalgouverneurs im besetzten Polen hervorzurufen. Der enthusiastische Brief Franks an Wiechert anlässlich des Beststellers „Das einfache Leben“, der vom Verf. als „kurioser Brief“ (298) leichtfertig abgetan wurde, ist für den Krakauer Statthalter Hitlers durchaus verständlich. Abgesehen vom gewissermaßen musischen Ehrgeiz Hans Franks, drückt diese Bewunderung für die „Handvoll Deutsche als Führungsschicht in einem Raum mit 14,5 Millionen Fremdvölkischer" eine eindeutige Parallele aus: so wie der Wiechertsche Held von Orla in der (?) Einsamkeit seiner Wälder agiert, so haben die wenigen Vertreter der deutschen Führungsschicht in dem stummen Wald von 14,5 Millionen auch eine fest umrissene Aufgabe zu erfüllen und eine Stärkung kann ihnen nur von Seiten der deutschen Kultur und der deutschen Religiosität zukommen. Im Verhältnis zum Frühwerk ändert sich hier eigentlich nur wenig: der Antiurbanismus, die Waldherrlichkeit, das Lob des einsamen heroischen Helden bilden das Gemeinsame mit dem „,Totenwolf“. Das nun präsente christreligiöse Element verstärkt nur die Popularität des Beststellers beim bürgerlichen Publikum und sichert dem Autor das mehr als verständliche Wohlwollen der politischen Randinstanzen wie Dr. Hans Frank. Als Beispiel der Inkompatibilität des Romans mit der NS-Ideologie wird mit Recht die Rezension von Bernhard Payr (300) genannt, der sein Elaborat im Rosenbergschen Lektoren-Brief veröffentlichte. Der Verlag Langen-Müller (seit 1936 auch offizieller Verlag der DAF), der mit dem sofort in mehrere Sprachen übersetzten Werk ein gutes Geschäft machte, war sich der Bedeutungslosigkeit der Rosenbergschen Stelle bewusst, die eigentlich nichts zu sagen hatte, weil das mächtige Ministerium für Propaganda und Volksaufklärung mit seiner Agenda, der RSK, die eigentlichen Entscheidungen traf. (Was mich ein wenig wundert ist die Tatsache, dass der Verf. nicht die RSK-Akte Wiecherts im Bundesarchiv finden konnte oder wollte? Abgesehen von dem fehlenden Versuch, das durchaus existierende Verlagsarchiv des Langen-Müller-Verlages zu konsultieren ${ }^{1}$ ). Das Markenzeichen der Poetik der Werke der inneren Emigration war

${ }^{1}$ In Bezug auf den Erfolg von Ernst Wiechert kann man feststellen, dass er wirklich mit der Übergabe seiner Werke an den Verlag Langen-Müller in Verbindung stand. 1931 wurde Wiechert durch Gustav Petzold auf Schloss Osterstein entdeckt, Diese für Wiechert sehr bequeme Situation, in einem Verlag der DAF veröffentlichen zu können, war zugleich mit seinem internationalen Erfolg verbunden. Der Verf. gibt nach Plesske auf S. 138 seines Buches die großen Summen, die Wiechert in den Jahren 1939 und 1940 verdiente, verschweigt aber die Werbetätigkeit des Verlages, die zur 
gerade ihre verschwommene agile konservative Haltung, die unterschiedliche ideologische Angebote zu formulieren und zu befriedigen imstande war. Bei diesen Beurteilungen lässt sich der Verf. durch eine Kohärenzvorstellung von der Kulturpolitik des Dritten Reiches leiten. Es ist freilich schwierig, sich im Labyrinth von diversen Interessen, Institutionen, regionalen Bedingtheiten zurechtzufinden. Auf jeden Fall würde eine präzise Beschreibung der Verlagspolitik im Verlag Langen-Müller mit Bezug auf Wiechert und eine sorgfältige Rezeptionsanalyse des Romans durch diverse Autoren und Zeitschriften gut tun, statt nach ideologischen Verdachtsmomenten zu suchen und die ziemlich verschwommenen, weil ästhetisch bedingten Aussagen, zu ideologischen Deklarationen hochzustilisieren.

Zu tadeln sind manche Verhaltensweisen des Verf., die als „nicht elegant“ bis „,schäbig“ einzustufen sind. Nicht elegant ist bestimmt die Ignorierung der Hinweise auf diejenigen Stellen in der Forschungsliteratur, die bereits die vom Verf. erörterten Tatsachen besprachen, wie zum Beispiel Hinweise auf die „Musterrezension“ von Payr: Der Verf. stellt zwar fest, dass sich ein Hinweis darauf in der neueren Arbeit von Klapper befindet (110), missachtet aber die Tatsache, dass in einer wesentlich früheren Studie, nämlich der von Barbian, die dem Verf. durchaus bekannt ist, eine Erwähnung dieses Sachverhaltes stattfand. Bei der zweimaligen und sehr breiten Zitation der Musterbesprechung (kein Wunder, sie sollte ja als Paradebeispiel der Ferne Wiecherts zum Nationalsozialismus fungieren, 287, 300) findet man aber keine Erwähnung, dass die Sache in der Forschungsliteratur hinlänglich berücksichtigt wurde. Das ist nicht elegant. Schäbig dagegen ist, dass der Verf. die Arbeit seiner Breslauer Kollegin Anna Gajdis über die ostpreußische Literatur, darunter über Wiechert, mit keinem Wort erwähnt. ${ }^{2}$ Ebenfalls werden weder eine Monografie von Jan Rosłan aus dem Jahr $1992^{3}$ noch der kritische Aufsatz über Wiechert von Hartmut Eggert in Roczniki Humanistyczne der Katholischen Universität in Lublin (1994) erwähnt. ${ }^{4}$ Eine andere Nicht-Erwähnung ist dagegen mehr als markant: nämlich die der Studie Der ungeheure Verlust. Flucht und Vertreibung in der deutschsprachigen Belletristik der Nachkriegszeit von Louis Ferdinand Helbig aus dem Jahr 1996, in dem sich bedeutende Indizien auf die Rezeption Wiecherts durch die Kreise der „Heimatvertriebenen“ finden lassen. Dafür gebraucht der Verf. peinliche Heimatvertriebenenrhetorik und zitiert allen Ernstes Ergüsse der Heimatvertriebenen aus solchen Organen wie die „Storchenpost". Eine eingehendere Analyse der Schriften Wiecherts aus dieser Zeit (vor allem die der Jeromin-Kinder oder Missa sine nomine) würde bestimmt den „Konservatismus“ des Schriftstellers enthüllen sowie die Mechanismen einer frühen Kulturpolitik der Vertriebenen zumindest skizzieren müssen. Das darf aber nicht sein, da der Verfasser vom „Ein-

sofortigen Übersetzung des „Einfachen Lebens” in einige Fremdsprachen führte. Außerdem scheint der Verfasser zu meinen, es handelte sich auch nach 1932, also nach der Petzoldschen Fusion um zwei Verlage, was ein offensichtlicher Irrtum ist, siehe S. 138 der Arbeit von Gołaszewski. Zu der Fusion siehe: Andreas Mayer (1988): Die Verlagsfusion Langen-Müller. Zur Buchmarkt- und Kulturpolitik des Deutschnationalen Handlungs-Gehilfen-Verbandes (DHV) in der Endphase der Weimarer Republik. In: AGB 32, S. 1-271.

2 Anna Gajdis (2014): Odległa prowincja. Prusy Wschodnie w prozie pisarzy niemieckich (1866-1945). Wrocław.

3 Jan Rosłan (1992): Ernst Wiechert. Życie i dzieło. Olsztyn.

${ }^{4}$ Hartmut Eggert (1994): Schwierigkeiten mit Ernst Wiechert. Innerlichkeit und innere Emigration. In: Roczniki Humanistyczne KUL 42, H. 5., S. 5-22. 
fachen Leben“ ja weder konservativ noch heimatgebunden im Sinne der Heimatideologie der Vertriebenen war. Obwohl der Verf. die polnische Forschungsliteratur grundsätzlich ignoriert, macht er eine Ausnahme: in der Bibliographie gibt er den Aufsatz von Hubert Orłowski an: Ernst Wiechert a tradycje konserwatywne i emigracja wewnętrzna (Ernst Wiecherts Verhältnis zu den konservativen Traditionen und die innere Emigration 2003). Hätte er den Aufsatz übrigens wirklich gelesen, dann würden ihm viele oben angedeuteten Verwechslungen erspart bleiben. Abgesehen davon ist für mich die Dokumentierung der im sehr umfangreichen (S. 442-579) Anhang veröffentlichten Lebenszeugnisse Wiecherts nicht plausibel genug. Nach der tabellarischen Zusammenstellung der biographischen Daten sowie der (wertlosen) Aufzeichnung der Erscheinungsjahre der Erstausgaben (das bekommt der Leser anstatt einer ordentlichen Bibliographie), folgt zuerst, auf S. 441-452 eine Reihe von Übersetzungen aus der nordischen Presse (Dänemark, Norwegen) über eine Dichterreise Wiecherts. In welcher Mappe und in welchem Archiv der Verf. das gefunden hat, kann man nur ahnen. Dann kommen drei umfangreiche Briefe aus den Jahren 1948 und 1949 mit Strohdtmann und O. Seidlin, ebenfalls aus einer geheimnisvollen Mappe in einem geheimnisvollen Archiv. Danach endlich als Sahnehäubchen auf der Torte der Segen aller Wiechert-Apologeten, das hier mehrmals erwähnte Gutachten der Rosenbergschen Stelle, dessen Herkunft sich im ehemaligen (weil 2016 geschlossenen) Königsberg-Museum in Duisburg feststellen lässt. Kein Wort über den Aufbau des Nachlasses, über die Art der Aufbewahrung, über die Auswahl (?) der hier veröffentlichten Dokumente. Manches wird aus dem Internet abgedruckt (S. 549-550), manches wird schlichtweg falsch beschrieben (S. 520 - nicht der Brief des Reichsführers, sondern eines Beamten in seiner Adjutantur) usw. usf. Die Materialien sind zum Teil sehr interessant, vor allem der Briefwechsel mit Tucholski (486-498), obwohl wiederum keine Information zu dieser Quelle geliefert wird. Es ist schade, dass der Verf. (lies: mechanisch) den Anhang so nachlässig veröffentlichte. Bei genauerem Hinsehen, d.h. bei einer Kommentierung könnte man wirklich bedeutende Aufschlüsse zu den Kontakten und Haltungen Wiecherts gewinnen. $\mathrm{Zu}$ den weiteren Schäbigkeiten des Verf. gehört auch die Behandlung der für ihn prominenten Forschungsliteratur. Zum Beispiel: Das Kapitel 10. Der Weg ins Exil: 1945- (k)eine Stunde Null im literarischen Werk Ernst Wiecherts (S. 349-365) wird mit der Fußnote folgendermaßen kommentiert: „Dieses Kapitel stützt sich teilweise auf meinen Artikel: Gołaszewski, Marcin, Kardach, Magdalena: Macht und Kultur unter dem Einfluss der Kulturpolitik nach und vor 1945 am Beispiel des literarischen Werkes von Ernst Wiechert [...].“ Abgesehen davon, dass der erste Teil des Artikels, wohl geschrieben von Magdalena Kardach, die im Unterschied zu dem Verf. zum Beispiel keineswegs die Herkunft mancher Schriften der Sekundärliteratur aus dem Umkreis der Vertriebenenverbände verschleiert, sich wohltuend von der forcierten Entwicklungsthese des Verf. unterscheidet, ist dazu zu sagen, dass sich der genannte Artikel im zweiten Teil sowie das 10. Kapitel der Monographie auf den nicht ausdrücklich genannten Aufsatz von Leonore Krenzlin stützen: Zwischen allen Stühlen. Ernst Wiechert in der politischen Öffentlichkeit 1933 bis 1947. In: Von bleibenden Dingen. Über Ernst Wiechert und sein Werk. Hg. v. Bärbel Beutner und Hans-Martin Pleßke, Frankfurt am Main 2002, S. 21-41. Eine weitere Peinlichkeit lässt sich durch das Zitat aus einer Notiz auf der Rückseite des Briefes Hans Grimms an Wiechert ablesen. Sowohl im genannten Aufsatz Gołaszewskis als auch in seinem Buch, S. 354, Fn. 923, wird eine Transkription des Briefes dargeboten und die Adresse, nämlich das Deutsche Literaturarchiv in Marbach, angegeben. Somit entsteht der Eindruck, der Verf. 
ist nach Marbach gefahren, hat im Nachlass Grimms gestöbert, den Brief gefunden, ihn transkribiert und das Ergebnis dem Leser mitgeteilt. Leider ist das nicht der Fall, weil der besagte Brief und genauer das in Frage kommende Zitat bereits in einer anderen Arbeit zitiert wird. (Manfred Franke: Jenseits der Wälder. Der Schriftsteller Ernst Wiechert als politischer Redner und Autor. Köln 2003, S. 90)

Wohin man schaut findet man Meinungen aus zweiter Hand, Ergebnisse, die gar keine sind, weil sie nicht aus den vorangehenden Analysen resultieren, dazu noch die hier geschilderten „Strategien“ der wissenschaftlichen Schäbigkeit, in die der Adept ziemlich viel Kraft und Zeit investierte, statt schlüssige Fragestellungen zu entwerfen, methodische Prägnanz zu demonstrieren, zu neuen Erkenntnissen zu kommen. In Summa: die vom Verf. selbstlobend gepriesene erste komplexe Monografie über den Schriftsteller Ernst Wiechert ist eine Leistung aus zweiter Hand, die vor dem Hintergrund der existierenden umfangreichen Forschungsliteratur gar nichts oder nur in geringem Maße zur Erkenntnis des Dichters beiträgt. Die Ursachen dafür sind: mangelnde Auseinandersetzung mit der Forschungsliteratur (keine Hinweise auf die interessengeleiteten Forschungsgruppen wie die Ernst-Wiechert-Gesellschaft), mangelnde Kenntnis der ideologisch-historischen Zusammenhänge (keine Kenntnis des Konservatismus, der konservativen Revolution, der Heimatschutzbewegung und der Lebensreformbewegung um die Jahrhundertwende), keine Kenntnis des literaturtheoretischen Instrumentariums (fataler Biographismus der gesamten Abhandlung) und der ins Lächerliche ausgeprägte Wille zur Durchsetzung des eigenen kolossalen Projektes, nämlich der ersten komplexen Monografie zu Ernst Wiechert.

\author{
Wojciech Kunicki \\ (Universität Wrocław)
} ORCID: 0000-0003-4005-0769

Wojciech Kunicki, Uniwersytet Wrocławski, Instytut Filologii Germańskiej, Pl. Nankiera 15b, 50-140 Wrocław, Polen, E-Mail: wojciech.kunicki@uwr.edu.pl

Received: 22.09.2018, accepted: 8.04.2019

\title{
Polnische Spuren in Deutschland im Blickfeld eines Lesebuchlexikons
}

\author{
Dieter Bingen, Andrzej Kaluza, Basil Kerski, Peter Oliver Loew (Hrsg.): \\ Polnische Spuren in Deutschland. Ein Lesebuchlexikon. Bundeszentrale für \\ politische Bildung, Bonn 2018, 447 S.
}

DOI: $10.19195 / 0435-5865.144 .27$

„Als vor 50 Jahren die ,Ostdenkschrift‘ der Evangelischen Kirche und der Brief der pol-
nischen Bischöfe an ihre deutschen Amtsbrüder veröffentlicht wurden, bestand zwischen
beiden Gesellschaften weitgehende Sprachlosigkeit, mussten zunächst einmal Ebenen des
Gesprächs gefunden werden. Heute gibt es Kontakte in vielen Bereichen [...]. Nicht nur 